\title{
Short-term memory and sentence processing: Evidence from neuropsychology
}

\author{
RANDI C. MARTIN \\ Rice University, Houston, Texas
}

\begin{abstract}
Traditional models of memory assume that short-term memory, as measured by memory span, plays an important role in linguistic processing and the learning of verbal information. Contradicting this view are findings from a brain-damaged patient, E.A., who, despite a verbal memory span of about two items, demonstrated normal sentence comprehension in a variety of tasks. She was, however, impaired whenever verbatim phonological information had to be maintained or learned. These results and those from other patients with reduced span suggest that the phonological storage capacity that is critical to memory span plays only a limited role in language processing, specifically in the maintenance and learning of phonological forms. Implications for models of short-term memory are discussed. It is argued that short-term memory should be seen as deriving from the processing and retentive capacities of language processing modules, with span tasks drawing on only a subset of these modules.
\end{abstract}

Neuropsychological findings from the 1960 s had important implications for the models of short-term memory that were being developed at the time. Neuropsychological findings that have accumulated since then also have a bearing on theories of short-term memory, sometimes providing confirmation of theories developed from studies of normal subjects, sometimes providing a challenge to these theories, and often providing insights that lead to new directions for research. This paper provides a review of the results of some of my own and other researchers' studies on brain-damaged patients that have a bearing on certain aspects of short-term memory-specifically, the role of short-term memory in language processing. This review addresses the fundamental question of whether the short-term memory that is tapped by memory span tasks plays the general role in verbal learning and language processing that is assumed by traditional memory models.

Returning to the neuropsychological findings from the 1960s, striking findings were obtained for the well-known cases of H.M. (Milner, 1966) and K.F. (Warrington \& Shallice, 1969). H.M. showed a normal digit span but a severe impairment of long-term retention of new information. K.F. showed the contrasting pattern of a very restricted digit span (he could recall only one item reliably) but a preserved ability to learn new information. These findings were interpreted as consistent with what Murdock (1974) originally termed the "modal model" of memory (e.g., Atkinson \& Shiffrin, 1968; Waugh \& Nor-

\footnotetext{
The preparation of this manuscript was supported by National Institutes of Health Grant DC 00218 to R. C. Martin at Rice University. This paper was presented in the symposium titled "Short-Term Memory: Where Do We Stand?' ' at the the Annual Meeting of the Psychonomic Society, San Francisco, November 1991. Correspondence should be addressed to R. C. Martin, Psychology Department, Rice University, P.O. Box 1892, Houston, TX 77251 (e-mail: rmartina@ricevml. rice.edu).
}

man, 1965), which postulated separate short- and longterm stores. That is, H.M. had a preserved short-term memory and disrupted long-term memory, whereas K.F. had the reverse dissociation. The findings for K.F. posed some difficulty for the modal model, however, since the modal model assumed that information had to be retained in short-term memory to be transferred to long-term memory. Given that K.F. showed normal list and pairedassociate learning (Warrington \& Shallice, 1969), it seemed that his defective short-term memory did not impair his ability to learn new information. Another problem for the modal model was that short-term memory was seen as a working memory system used for thinking and problem solving, and presumably, language comprehension as well. However, K.F. did not show the severe intellectual deficit that might be predicted from a one-item working memory capacity, but showed at least fairly good language comprehension.

A possible solution to these difficulties for the modal model is offered by Baddeley's working memory model (Baddeley, 1986). According to this model, short-term serial recall for word lists is supported primarily by a special buffer system, termed the articulatory loop, rather than by the totality of working memory. In Baddeley's model, working memory consists of a central executive system and several passive storage, or slave, systems. The central executive is assumed to be an attentional control system used to supervise the activity of all the components of working memory and integrate the information retained in slave systems. The slave systems are assumed to be more passive storage systems used to retain information in particular coding formats. The articulatory loop is one of these slave systems, argued to consist of a phonological storage buffer and an articulatory rehearsal mechanism. These assumptions about the nature of the articulatory loop are supported by several findings from span 
tasks, including the detrimental effects of phonological similarity and word length on span, and the decrement in span performance seen with articulatory suppression (e.g., Baddeley, Lewis, \& Vallar, 1984; Baddeley, Thomson, \& Buchanan, 1975; Conrad, 1964). Within Baddeley's working memory model, K.F. could be argued to have a disruption of the articulatory loop component of working memory, but not the other components. An implication from the findings for K.F. would be, then, that the articulatory loop is not necessary for normal longterm retention of verbal materials as tested by paired associate and list recall paradigms.

\section{SHORT-TERM MEMORY AND SYNTACTIC PROCESSING}

The question then arises as to the primary function of the articulatory loop outside of supporting the performance of traditional short-term memory tasks such as memory span. A possible answer is that the articulatory loop is used to hold verbal information during both oral and written sentence comprehension. After all, during sentence comprehension it is necessary to integrate early words with later words to arrive at an interpretation of the sentence as a whole, and some means for retaining the early information should be important. Furthermore, word order is important for sentence comprehension, especially for the analysis of syntactic structure, and the retention of order information appears to be an important function of the articulatory loop (Wickelgren, 1965). This suggestion that verbal short-term memory is important for language comprehension has been made by several authors. For example, Crowder (1982) suggested that "deep down, many of us believe that the main role of short-term memory must surely be in the perception and comprehension of language" (p. 309 ), and "the role of short-term memory may really be in deciphering syntax" (p. 316).

The study of the relation of short-term memory and sentence processing has been an active area of neuropsychological research. The patients that have attracted the most attention are those such as K.F. for whom reduced memory span is the most striking deficit. A number of patients have been identified whose short-term memory deficit appears due to a phonological storage deficit rather than to difficulty with rehearsal. As reviewed by Shallice and Vallar (1990), these patients show better immediate recall with visual presentation than with auditory presentation (the reverse of the normal pattern), no word length effects, phonological similarity effects for auditory but not visual presentation, and reduced or absent recency effects. In addition, many of the patients have been shown to perform poorly on recognition probe tasks and on matching span tasks (i.e., determining whether two lists of numbers are the same or differ in the order of two adjacent items). Several of these features are not consistent with a rehearsal deficit. For example, normal subjects under articulatory suppression (which is presumed to disrupt articulatory rehearsal) do not show a reverse modality ef- fect, but instead show a larger auditory advantage (Levy, 1971). Also, the patients' poor performance on memory probe and matching span tasks could not be attributed to a rehearsal deficit since articulatory rehearsal appears not to be involved in these tasks (Chase, 1976; Monsell, 1987). Finally, normal subjects under suppression show larger spans than do these patients (Baddeley et al., 1975).

The results of the study of brain-damaged patient P.V., who showed the short-term memory pattern described by Shallice and Vallar (1990), were used by Baddeley and colleagues to support the division of the articulatory loop into a passive phonological storage capacity and a more active articulatory rehearsal process (Baddeley, 1986; Vallar \& Baddeley, 1984a). According to this formulation, P.V. and other patients showing this short-term memory pattern have a disruption of the phonological storage component. The reverse modality effect demonstrated by these patients can be accounted for if it is assumed that the recall of visually presented items can be supported, at least partially, by a visual storage capacity that is not damaged in these patients. Recall of auditorily presented items, on the other hand, is assumed to depend on the phonological store. Damage to the phonological store can account for the absence of phonological similarity and word length effects for visual presentation on the grounds that, unlike the case for normal subjects, there is no advantage to the patients to convert a visual code to a phonological code. The presence of a phonological similarity effect for auditory presentation might seem to contradict the phonological store deficit; however, the auditory effect can be accounted for by assuming that although the phonological store capacity is reduced it is not zero. If it is argued, though, that these patients retain some reduced phonological storage capacity, the absence of a word length effect for auditory presentation is somewhat more difficult to explain. Vallar and Baddeley (1984a) suggested that these patients might not opt to use rehearsal as a strategy because their very reduced phonological storage capacity makes such a strategy of little use. Alternatively, it is possible that these patients do rehearse the little information that they can retain in a phonological form (Warrington \& Shallice, 1972), but the rehearsal of larger groups of items is necessary to detect a word length effect.

Studies carried out during the late 1970 s and early 1980 s seemed supportive of a connection between reduced phonological storage capacity and difficulty carrying out syntactic analyses. Saffran and Marin (1975) reported results from a patient with evidence of a phonological short-term memory deficit who was tested on the repetition of oneclause and two-clause active and passive sentences. In his repetitions, the patient had a tendency to transform reversible passives to actives (changing the meaning) and was more likely to do so for the two-clause sentences than for the one-clause sentences. (A reversible sentence is one in which the roles of agent and object could be reversed and the sentence would still make sense, as for "The girl pushed the boy.") Also, Caramazza, Basili, Koller, and Berndt (1981) reported a patient with a similar short-term 
memory deficit who showed difficulty with sentence comprehension on a sentence-picture matching task. When asked to choose a picture to match a reversible active or passive sentence or a reversible relative clause sentence (e.g., "The lion that the tiger chased was yellow'), he often chose a distractor picture that reversed the roles of agent and object. The data from both of these patients suggested that they had difficulty processing the syntactic structure of sentences, causing an impaired ability to assign role relationships in the sentences.

However, more recent studies have called into question this relation between phonological short-term storage and syntactic processing. My colleagues and I have studied a brain-damaged patient, E.A., extensively (Martin, 1987, 1990; Martin, Shelton, \& Yaffee, in press; Shelton, Martin, \& Yaffee, 1992). She shows the short-term memory pattern described above and has a span of about two words for auditory presentation and about three words for visual presentation. Figure 1 shows her serial recall of auditory and visual superspan letter lists. The superiority of recall for visual presentation is evident in the figure, as is the absence of a recency effect, at least with auditory presentation. Other results for E.A. have documented the phonological similarity and word length effects that are characteristic of a phonological storage deficit (Martin, 1987).

One test that was carried out on E.A.'s sentence processing abilities investigated her ability to detect gender mismatches between pronouns and their referents and to detect word order reversals. To the extent that words are retained in a phonological form in order to integrate earlier and later portions of a sentence, one might expect that the farther the pronoun from its referent, the more difficulty E.A. should have in making this integration. Similarly, if phonological short-term memory is needed to hold the order of words in a sentence, then E.A. should have difficulty detecting word order reversals, and this difficulty should be greater with more words in the sentence. Table 1 provides examples of the incorrect sentences that were used. (An equal number of similarly constructed correct sentences were also presented.) For the gender mismatch sentences, short, medium, and long sentences were used, with the distance between the pronoun and referent increasing with sentence length. For the word order reversal sentences, short and long sentences were used, with a greater distance between the reversed noun phrases for the long sentences. For both sentence types, the correct and incorrect sentences were presented in a random order, and E.A. and the control subjects were asked to judge whether the sentence was acceptable or anomalous.

As shown in Table 2, E.A. had no difficulty with the gender mismatch sentences, performing at least as well as the mean for the control subjects for all sentence lengths. For the word order reversal sentences, E.A.'s scores were below the mean for the control subjects, but within their range. These data indicate that E.A. is able to integrate information over long distances and process the order of words in a sentence, despite her very restricted memory span.

Unlike the patients reported by Caramazza et al. (1981) and Saffran and Marin (1975), E.A. was able to understand simple active and passive sentences and some more

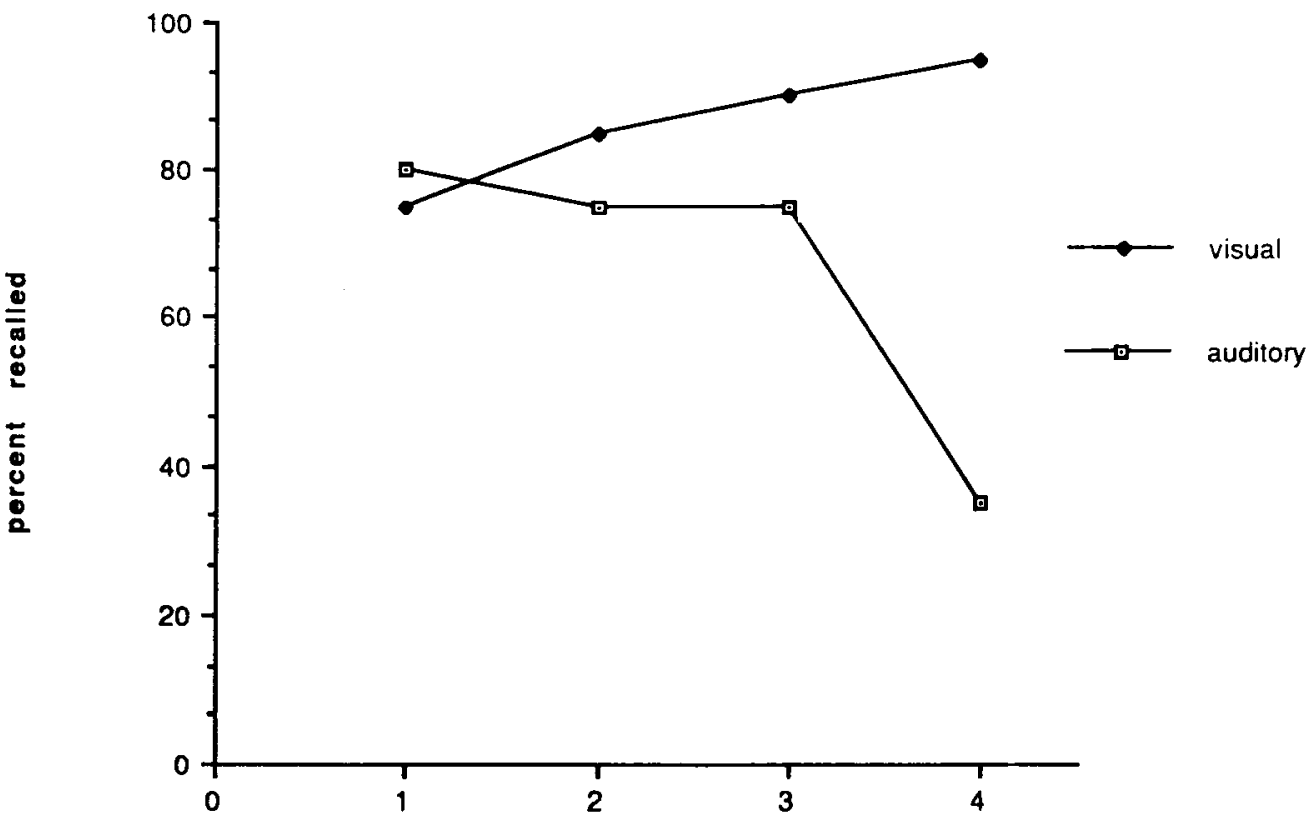

serial position

Figure 1. Percent of letters recalled by patient E.A. as a function of serial position at presentation. 
Table 1

Materials for Sentence Acceptability Judgments

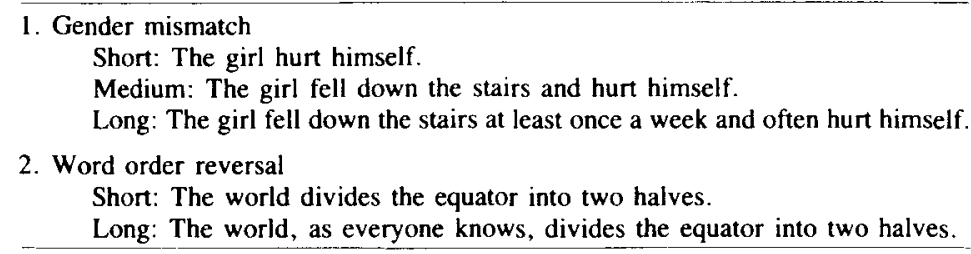

complex center-embedded relative clause sentences (Martin, 1987, 1990). Table 3 shows examples of sentence types that she was tested on using a sentence-picture matching task. For all sentence types, E.A. was asked to select from two pictures the one matching an auditorily presented sentence. For the one-clause active and passive sentences, the distractor picture reversed the role of agent and object. For the relative clause sentences, on half the trials, the distractor picture showed a reversal of agent and object with respect to the action verb, whereas on the other half, the distractor picture depicted the descriptive phrase as modifying the wrong person or object. Thus, both types of trials tested E. A.'s ability to analyze the syntactic structure of these sentences. All of the relative clause sentences had center-embedded constructionsthat is, where the head noun is separated from the main verb by the intervening relative clause. If the head noun had to be maintained in a phonological form for integration with the main verb, one might expect E.A. to have difficulty with all of the relative clause sentence types. The results for the sentence-picture matching task are also shown in Table 3. ${ }^{1}$ Normal control subjects scored $100 \%$ correct for all sentence types except for the type 4 relative clause sentences, where the control mean was $98 \%$

Table 2

Percent Correct for Sentence Acceptability for Patient E.A.

\begin{tabular}{lcccccc} 
& \multicolumn{3}{c}{ Gender Agreement } & & \multicolumn{2}{c}{ Word Order } \\
\cline { 2 - 3 } \cline { 6 - 7 } & Short & Medium & Long & & Short & Long \\
\hline E.A. & 90 & 90 & 90 & & 78 & 72 \\
$\begin{array}{l}\text { Control } \\
\begin{array}{l}\text { Mean } \\
\text { Range }\end{array}\end{array}$ & 91 & 88 & 87 & & 88 & 85 \\
\hline
\end{tabular}

Table 3

Syntactic Comprehension for Patient E.A.: Example Sentences and Percent Correct (Sentence-Picture Matching)

Percent Correct

$$
\text { One-Clause Reversible Sentences }
$$

Active: The dog chased the cat

Passive: The cat was chased by the dog.

\section{Center-Embedded Relative Clause Sentences}

1. The cat that was black chased the dog.

92

2. The cat that chased the dog was black.

92

3. The cat that was black was chased by the dog.

92

4. The cat that was chased by the dog was black. $\quad 58$

5. The cat that the dog chased was black.

42

Note-The data for the relative clause sentences were originally reported in Martin (1990). correct. E.A. performed very well on the active and passive sentences and on the first three types of relative clause sentences. She did have difficulty with the relative clause sentences that included a complex syntactic structure in the embedded clause-that is, the sentences with a passive in the embedded clause (type 4) and the sentences with an object relative form in the embedded clause (type 5).

Despite E.A.'s difficulty with the most complex relative clause sentences, these results demonstrated an impressive ability to carry out syntactic analyses for a patient with a span of about two items. We initially hypothesized that E.A.'s difficulty with the two relative clause sentence types could be attributed to her short-term memory deficit (Martin, 1987). However, it has been found that other patients with short-term memory deficits as severe as E.A.'s could understand sentences that were as complex as the ones on which E.A. failed. Table 4 provides references for these cases and examples of complex sentence types on which they performed normally. Both H.B. (Howard, 1989) and B.O. (Waters, Caplan, \& Hildebrandt, 1991) could comprehend normally a variety of complex syntactic constructions, including the centerembedded object relative clause sentences that caused so much difficulty for E.A. ${ }^{2}$

These findings of good syntactic processing in the face of severely reduced phonological retention capacity are consistent with "immediacy of processing" theories of sentence comprehension (Just \& Carpenter, 1987). Many studies with normal subjects support the view that as much processing as possible is carried out on each word as it is perceived. Several such studies have used "gardenpath" sentences, that is, sentences in which the reader is led to assume a particular syntactic structure or semantic interpretation of a sentence, which later proves incorrect (e.g., "Since Jay always jogs a mile seems like a short distance to him"; Frazier \& Rayner, 1982). Studies of the comprehension of garden-path sentences using eye movement monitoring have shown that subjects show very long gaze durations on the first word of a sentence that indicates that the developing interpretation is incorrect (Carpenter \& Daneman, 1981; Frazier \& Rayner, 1982). An effect on this word would not be expected if semantic and syntactic analysis lagged behind the processing of each word. Tyler and Marslen-Wilson and colleagues (MarslenWilson, Brown, \& Tyler, 1988; Marslen-Wilson \& Welsh, 1978; Tyler \& Marslen-Wilson, 1977) have obtained similar evidence for immediate syntactic and semantic interpretation for auditorily presented sentences. 
Table 4

Examples of Sentence Types Comprehended by Patients with Phonological Short-Term Memory Deficits

1. R.E. (Butterworth, Campbell, \& Howard, 1986)

e.g., "The bus is preceded by the train which the triangle is below."

2. H.B. (Howard, 1989)

e.g., "The boy that the girl pushed was tall."

3. B.O. (Waters, Caplan, \& Hildebrandt, 1991)

e.g., "The bear that the donkey kissed patted the goat."

"Patrick appears to a friend of Joe's to be eating."

Because of this immediacy of processing, there is no need to retain an exact phonological record of the input lexical items and their order. As each word is perceived, the auditory information is transformed into a phonological code, and the syntactic and semantic features of the word are retrieved. These features are used to derive the syntactic structure and semantic interpretation of the sentence up to that point. The order of the words as they are perceived also determines the syntactic structure that is assigned. It is this structure and the semantic interpretation derived from the combination of this structure with the meaning of the words that need to be retained, not the phonological record. Thus, with respect to the sentence processing tasks carried out with E.A., it is the analysis of syntactic structure and the interpretation of the semantic features and thematic roles that need to be retained. For example, for the gender mismatch task, it is the information that the referent of the pronoun is male or female and the structural information indicating which word is the referent that need to be retained, not the phonological form of the words that carried this information. The evidence from E.A. and the other patients with reduced phonological stores and preserved syntactic comprehension suggests that whatever capacities are needed to retain the developing syntactic structure and semantic interpretation, they are not equivalent to those tapped by span tasks.

\section{ALTERNATIVE ROLES FOR SHORT-TERM MEMORY IN LANGUAGE PROCESSING}

Thus, we are back to the question of the role of the phonological short-term memory that is tapped by span tasks. One finding consistently obtained is that patients such as E.A. show poor verbatim retention of sentences (Butterworth, Campbell, \& Howard, 1986; Friedrich, Martin, \& Kemper, 1985; Martin, 1990; Saffran \& Marin, 1975). When asked to repeat 30 sentences that were fairly long and had complex syntactic structures, E.A. repeated only one sentence verbatim (Martin et al., in press). (Of 3 control subjects tested on these materials, 2 repeated all of the sentences correctly, and the other made an error on one sentence.) Most of E.A.'s attempts at repetition were paraphrases that either preserved the meaning of the original sentence ( $50 \%$ of the sentences) or were only slightly different in meaning (10\% of the sentences). ${ }^{3}$ For example, for the sentence "After eating dinner, the man walked the dog," E.A. produced "After supper, the man took his dog for a walk," and for the sentence "Before calling her mother, the girl had a cup of tea," E.A. produced "The girl drank some hot tea before she went to talk to her mother." These responses suggest that although E.A. has understood the sentences, she is unable to reproduce the exact wording due to her inability to retain phonological information. It may not seem surprising that phonological memory appears to be involved in verbatim sentence repetition. Recently, however, Potter and Lombardi (1990) have argued, on the basis of results from normal subjects, that sentence repetition depends on regeneration of the sentence from its meaning, plus the incorporation of recently activated lexical items. They have suggested that the ordered auditory memory that is important in list retention does not play a role in sentence repetition. The sentence repetition data from E.A. and other patients with phonological short-term memory deficits indicate that phonological short-term memory does play at least some role in verbatim repetition.

Another plausible role for phonological storage is to provide a means of retaining a backup phonological representation of a sentence in case the initial, first-pass analysis proves incorrect and a second interpretation must be attempted. This possible role has received little attention in neuropsychological studies. In one attempt to address whether a phonological representation is used for secondpass processing (Martin, 1990), a sentence acceptability judgment task was administered to E.A. and some control subjects. In this task, some of the sentences were lexical garden-path sentences-that is, where preceding context biased the interpretation of an ambiguous word in one direction, but later context indicated that the other meaning of the word was the appropriate one. For example, one of the garden-path sentences was "The lawyer decided to take the case because it was large enough to hold all of his papers." To verify that the preceding context did in fact bias the inappropriate meaning of the ambiguous word (i.e., "case" in this example), we provided a group of undergraduate subjects with the sentences up to the ambiguous word and asked them to complete the sentence. Only sentences for which $75 \%$ or more of the completions indicated that subjects had interpreted the ambiguous word in the manner intended were used as stimuli. The garden-path sentences were presented mixed with sensible sentences that were not garden-path sentences and anomalous sentences such as "Sue ran past the grove where she had her checking account." The sentences were presented auditorily, and subjects were instructed to judge whether the sentences made sense. They were warned that some of the sentences might seem nonsensical at first but would seem sensible on second thought. Practice sentences, including some garden-path sentences, were administered with feedback. As reported in Martin (1990), E.A. performed at the mean of the age-matched control subjects for the sensible sentences (100\% correct) and garden-path sentences (80\% correct). On the anomalous sentences, she scored $80 \%$ correct, which was below the mean but within the range of the control subjects (mean 
$=93 \%$, range $=80 \%$ to $100 \%$ correct). Consequently, these data do not support the notion that a phonological record is needed for reinterpretation. However, more extensive testing should be carried out to examine syntactic garden-path sentences as well as lexical garden path sentences.

Another possible role for a backup phonological record would be in cases where, for auditorily presented sentences, processing cannot keep pace with the input. Difficulty processing the input might occur for a variety of reasons. For example, infrequent or mispronounced words or background noise might delay the access of the semantic and syntactic features of these words until later context disambiguates these words. A related, but somewhat different, situation might arise where one is attending to one of two auditory messages being spoken simultaneously by two speakers. In either case, the listener might have to hold information in a phonological form awaiting subsequent processing. Currently, there is little strong evidence that bears on whether the phonological store that is tapped by span tasks plays this role of maintaining auditory-verbal input until it can be fully processed (but see Romani, in press, for some relevant data).

Up to this point, these suggestions assign the phonological store to a subsidiary role in sentence processing. It is possible that the phonological store plays a more central role in language acquisition. One possibility is that the maintenance of words in a phonological form might be more important for children than for adults because children's semantic and syntactic processing abilities might be slower. That is, immediacy of processing would not apply to children who are just acquiring the syntactic rules of the language (Martin, 1990). Another possible role for short-term phonological retention in acquisition is in the learning of new phonological forms-that is, in the acquisition of new vocabulary. Baddeley, Papagno, and Vallar (1988) showed that their patient, P.V., was able to learn paired associates at a normal level when the words were familiar to her. However, she was completely unable to learn to produce a foreign translation of a word when given the word in her native language. We recently obtained a similar result when we attempted to teach E.A. eight pairs of English-Spanish equivalents. Half of the Spanish words had one syllable, and half had two syllables. E. A. could repeat the one-syllable Spanish words correctly, but had to be given several presentations of the two-syllable words before being able to repeat them correctly. ${ }^{4}$ In the paired associate task, after eight trials through the eight pairs, she could not produce a single Spanish word correctly when given the English word.

E.A.'s difficulty in repeating the Spanish words led us to further investigate her ability to repeat individual words and nonwords. For known (English) words, she repeated $98 \%$ of one-syllable words, $95 \%$ of two-syllable words, but only $75 \%$ of three- to five-syllable words. For nonwords, she repeated $79 \%$ of one-syllable nonwords and only $47 \%$ of two-syllable nonwords. Within the one syllable nonwords, she repeated $90 \%$ that had three phonemes and $73 \%$ that had four phonemes. Thus, for nonwords, there was a strong effect of the amount of phonological information that had to be retained. Her ability to retain more phonological information for words than for nonwords suggests a lexical and/or semantic contribution to single-word and word-list repetition (Hulme, Maughan, \& Brown, 1991; Martin et al., in press). ${ }^{5}$

E.A.'s difficulty in repeating nonwords might seem to be a logical consequence of damage to a system used to retain phonological information. It is more surprising that there may be a causal relation between poor retention of phonological information in the short-term and an impaired ability to establish long-term phonological representations for new words, as suggested by Baddeley et al.'s (1988) results. As indicated by Baddeley et al., this connection fits well with the assumptions of the modal model in which information had to be retained in shortterm memory in order to be transferred to long-term memory. However, unlike the case in the modal model, the capacity for phonological retention is only one aspect of short-term memory and the difficulty in establishing a long-term representation is specifically for phonological information, not for other types of information. Although these suggestions about a connection between short- and long-term phonological retention are intriguing, some caution must be exercised, since few patients with phonological short-term memory deficits have been examined for their long-term learning of phonological information. It is possible that other patients might be found who could establish long-term traces, at least for new words they could repeat. It should be noted, though, that Gathercole and Baddeley $(1989,1990)$ have found results consistent with a connection between the short- and long-term retention of phonological information in studies of vocabulary acquisition in children. They have found a correlation between children's nonword repetition ability and vocabulary size. This correlation persists even when the correlation is between nonword repetition ability at age 4 with vocabulary at age 5 , with vocabulary at age 4 partialed out (Gathercole \& Baddeley, 1989).

\section{CONCLUSIONS}

These results from E.A. and those from other patients with reduced span suggest that the phonological storage capacity that is critical to memory span does not serve as a general capacity for linguistic processing nor as a capacity that is necessary for learning associations between familiar words. This conclusion is warranted on the basis of E.A.'s normal performance on detection of gender mismatches and word order reversals, her ability to comprehend garden-path sentences, and her ability to repeat the gist of sentences. Also supporting this conclusion are the results from other patients with phonological storage deficits who show excellent comprehension of complex syntactic structures and a normal ability to learn new associations between known words (Baddeley et al., 1988; Butterworth et al., 1986; Howard, 1989; Warrington \& 
Shallice, 1969; Waters et al., 1991). Rather than serving as a general linguistic working memory, it appears that the phonological store plays a role limited to the maintenance and learning of specifically phonological information. This conclusion is supported by E.A.'s inability to repeat sentences verbatim, her difficulty in repeating nonwords and multisyllable words, and her inability to learn new phonological forms. Other patients with phonological storage deficits have also been reported who show the same deficits (Baddeley et al., 1988; Butterworth et al., 1986; Saffran \& Marin, 1975).

What are the implications of these neuropsychological findings for models of short-term memory? The findings on sentence processing suggest capacities for the retention of semantic and syntactic information that are separate from the capacities used to retain phonological information. If we return to Baddeley's working memory model, there appears to be no obvious place where such information is retained. Even with regard to span tasks, there is evidence for separate lexical and phonological contributions, but there is no obvious role for lexical representations in the articulatory loop. Thus, although this model allowed for separate storage for at least some kinds of information, further distinctions are needed.

Schneider and Detweiler (1987) have proposed a model that might be seen as an expanded version of Baddeley's approach, in which there are many modules dedicated to different types of information. However, rather than being passive stores, these modules carry out specific types of processing, such as auditory, phonological, lexical, and semantic processing. (A syntactic processing module should be added to the list to encompass sentence comprehension.) In this model, each processing module has dedicated storage capacity. Memory span can be seen within this model as deriving from the activity and storage capacity of those processing modules involved in certain aspects of language comprehension: speech perception and word recognition. In a sense, verbal memory span tasks involving the retention of random word lists can be seen as language processing tasks tapping only a subset of those processes typically involved in language comprehension (syntactic processing and elaborative semantic interpretation are left out). Short-term memory is closely tied to language processing in such an approach; however, to the extent that buffer storage is separable from processing mechanisms, processing is not identical with storage. Such a separation seems warranted by findings from patients who have demonstrated dissociations between speech perception and phonological retention-that is, excellent speech perception abilities and very poor phonological retention (Campbell \& Butterworth, 1985; Vallar \& Baddeley, 1984a, 1984b). Although E.A. does show a mild speech perception deficit, other patients with similar speech perception deficits show normal short-term memory (Martin \& Breedin, in press). Thus, in contrast to the assumptions of a levels of processing approach (Craik \& Lockhart, 1972), a distinction must be made between processing quality and the duration of the representations that result. Such an approach to verbal short- term memory that emphasizes the recruitment of language modules suggests that the initial question that was posed about the role of short-term memory in language processing should be reversed. Instead of asking what short-term memory has to do with language processing, we should ask what language processing has to do with short-term memory. That is, rather than seeing short-term memory as a separate system that is drawn upon in language processing, verbal short-term memory should be seen as deriving from the procedures and retentive capacities of language processing modules.

As I hope has become evident in this review, findings in the neuropsychological literature show some striking dissociations between short-term memory and language processing, and some intriguing associations as well. ${ }^{6}$ Not only are these findings of intrinsic interest, they provide constraints on what a theory of short-term retention and language processing should be like. Any adequate theory should be called upon to encompass both the findings from normal subjects and those from neuropsychological studies.

\section{REFERENCES}

Atkinson, R. C., \& Shiffrin, R. M. (1968). Human memory: A proposed system and its control processes. In K. W. Spence \& J. T. Spence (Eds.), The psychology of leaming and memory: Advances in research and theory (Vol. 2, pp. 89-195). New York: Academic Press.

Baddeley, A. D. (1986), Working memory. Oxford: Clarendon Press.

Baddeley, A. D., Lewis, V. J., \& Vallar, G. (1984). Exploring the articulatory loop. Quarterly Joumal of Experimental Psychology, 36A, 233-252

Baddeley, A. D., Papagno, C., \& Vallar, G. (1988). When longterm learning depends on short-term storage. Joumal of Memory \& Language, 27, 586-595.

Baddeley, A. D., Thomson, N., \& Buchanan, M. (1975). Word length and the structure of short-term memory. Journal of Verbal Learning \& Verbal Behavior, 15, 575-589.

Butterworth, B., Campbell, R., \& Howard, D. (1986). The uses of short-term memory: A case study. Quarterly Journal of Experimental Psychology, 38A, 705-737

Campaell, R., \& Butterworth, B. (1985). Phonological dyslexia and dysgraphia in a highly literate subject: A developmental case with as sociated deficits of phonemic processing and awareness. Quarterly Journal of Experimental Psychology, 37A, 435-475.

Caramazza, A., Basili, A. G., Koller, J., \& Berndt, R. S. (1981). An investigation of repetition and language processing in a case of conduction aphasia. Brain \& Language, 14, 235-271.

Carpenter, P. A., \& Daneman, M. (1981). Lexical retrieval and error recovery in reading: A model based on eye fixations. Journal of Verbal Learning \& Verbal Behavior, 20, 137-160.

CHASE, W. (1976). Does memory scanning involve implicit speech? In S. Dornic (Ed.), Attention and performance (Vol. 6, pp. 607-629). London: Erlbaum.

Conrad, R. (1964). Acoustic confusion in immediate memory. British Journal of Psychology, 55, 75-84.

Craik, F. I. M., Lockhart, R. S. (1972). Levels of processing: A framework for memory research. Journal of Verbal Learning \& Verbal Behavior, 11, 671-684

Crowder. R. (1982). The demise of short-term memory. Acta Psychologica, 50, 291-323.

Frazier, L., \& RAYNER, K. (1982). Making and correcting errors during sentence comprehension: Eye movements in the analysis of structurally ambiguous sentences. Cognitive Psychology, 14, 178-210.

Friedrich, F., Martin, R. C., Kemper, S. (1985). Consequences of a phonological coding deficit on sentence processing. Cognitive Neuropsychology, 2, 385-412. 
Gathercole, S. E., \& Baddeley, A. D. (1989). Evaluation of the role of phonological STM in the development of vocabulary in children: A longitudinal study. Journal of Memory \& Language, 28, 200-213.

Gathercole, S. E., \& Baddeley, A. D. (1990). Phonological memory deficits in language disordered children: Is there a causal connection? Journal of Memory \& Language, 29, 336-360.

HowarD, D. (1989, July). Does a short-term memory deficit cause impaired sentence comprehension? Paper presented at the International Conference on Cognitive Neuropsychology, Harrogate, U.K.

Howard, D., FrankLin, S. (1990). Memory without rehearsal. In G. Vallar \& T. Shallice (Eds.), Neuropsychological impairments of short-term memory (pp. 287-318). Cambridge: Cambridge University Press.

Hulme, C., Maughan, S., \& Brown, G. (1991). Memory for familiar and unfamiliar words: Evidence for a long-term memory contribution to short-term span. Journal of Memory \& Language, 30, 685-701.

JUST, M. A., \& CARPENTER, P. A. (1987). The psychology of reading and language comprehension. Newton, MA: Allyn \& Bacon.

LEVY, B. (1971). Role of articulation in auditory and visual short-term memory. Journal of Verbal Leaming \& Verbal Behavior, 10, 123-132.

Marslen-Wilson, W. D., Brown, C. M., \& Tyler, L. K. (1988). Lexical representations in spoken language comprehension. Language \& Cognitive Processes, 3, 1-16.

Marslen-Wilson, W. D., \& Welsh, A. (1978). Processing interactions and lexical access during word recognition in continuous speech. Cognitive Psychology, 10, 29-63.

MARTIN, R. C. (1987). Articulatory and phonological deficits in shortterm memory and their relation to syntactic processing. Brain \& Language, 32, 137-158.

MARTIN, R. C. (1990). Neuropsychological evidence on the role of shortterm memory in sentence processing. In G. Vallar \& T. Shallice (Eds.), Neuropsychological impairments of short-term memory (pp. 390-427). Cambridge: Cambridge University Press.

Martin, R. C., \& Breedin, S. (in press). Dissociations between speech perception and phonological short-term memory. Cognitive Neuropsychology.

Martin, R. C., Shelton, J. R., \& YafFe, L. S. (in press). Langauge processing and working memory: Neuropsychological evidence for separate phonological and semantic capacities. Journal of Memory \& Language.

MILNER, B. (1966). Amnesia following operation on the temporal lobes. In C. Whitty \& O. Zangwill (Eds.), Amnesia (pp. 109-133). London: Butterworth.

MoNsELL, S. (1987). On the relation between lexical input and output pathways for speech. In A. Allport, D. MacKay, W. Prinz, \& E. Scheerer (Eds.), Language perception and production: Relationships between listening, speaking, reading and writing (pp. 273-311). Orlando, FL: Academic Press.

MURDOCK, B. B., JR. (1974). Human memory: Theory and data. Hillsdale, NJ: Erlbaum

Potter, M. C., \& Lombardi, L. (1990). Regeneration in the shortterm recall of sentences. Joumal of Memory \& Language, 29, 633-654.

Romani, C. (1992). Are there distinct input and output buffers? Evidence from an aphasic patient with an impaired output buffer. Language \& Cognitive Processes, 7, 131-162.

RomanI, C. (in press). The role of phonological short-term memory in syntactic parsing: A case study. Language \& Cognitive Processes.

SAFfran, E. M., \& Marin, O. S. M. (1975). Immediate memory for word lists and sentences in a patient with deficient auditory short-term memory. Brain \& Language, 2, 420-433.

SCHNEIDER, W., DETWEILER, M. (1987). A connectionist/control architecture for working memory. In G. Bower (Ed.), The psychology of learming and motivation: Advances in research and theory (Vol. 21, pp. 54-119). New York: Academic Press.

Shallice, T., \& Vallar, G. (1990). The impairment of auditory-verbal short-term storage. In G. Vallar \& T. Shallice (Eds.), Neuropsychological impairments of short-term memory (pp. 11-53). Cambridge: Cambridge University Press.

Shelton, J., Martin, R. C., \& Yaffee, L. (1992). Investigating a verbal short-term memory deficit and its consequences for language processing. In D. Margolin (Ed.), Cognitive neuropsychology in clinical practice (pp. 131-167). New York: Cambridge University Press.

TYLER, L. K., MARSLEN-WILson, W. D. (1977). The on-line effects of semantic context on processing. Journal of Verbal Leaming \& Verbal Behavior, 16, 683-692.

VAlLAR, G., \& BADDELEY, A. D. (1984a). Fractionation of working memory: Neuropsychological evidence for a phonological short-term store. Journal of Verbal Learning \& Verbal Behavior, 23, 121-142.

Vallar, G., \& Baddeley, A. D. (1984b). Phonological short-term store, phonological processing and sentence comprehension: A neuropsychological case study. Cognitive Neuropsychology, 1, 121-141.

Warrington, E. K., Shallice, T. (1969). The selective impairment of auditory-verbal short-term memory. Brain, 92, 885-896.

Warrington, E. K., \& Shallice, T. (1972). Neuropsychological evidence of visual storage in short-term memory tasks. Quarterly Journal of Experimental Psychology, 24A, 30-40.

Waters, G., Caplan, D., \& Hildebrandt, N. (1991). On the structure of verbal short-term memory and its functional role in sentence comprehension: Evidence from neuropsychology. Cognitive Neuropsychology, 8, 81-126.

Waugh, N. C., Norman, D. A. (1965). Primary memory. Psychological Review, 72, 89-104.

WICKELGREN, W. (1965). Acoustic similarity and intrusion errors in short-term memory. Joumal of Experimental Psychology, 70, 102-108.

\section{NOTES}

1. The results for the relative clause sentences were taken from Martin (1990). The data for the active and passive sentences were obtained at the same time as the relative clause data but were not reported in that study.

2. Further testing with E.A. on these center-embedded relative clause sentences showed that she performed at chance with these constructions even when short-term memory demands were minimized-that is, when the sentences were presented visually with unlimited time for making the picture choice (Martin, 1990). Thus, it appears that E.A. has a mild syntactic deficit that affects her ability to analyze certain complex syntactic forms. Given the good performance of the patients in Table 4 on similar constructions, E.A.'s syntactic difficulties appear to be independent of her short-term memory deficit.

3. The remainder of her ertors consisted of sentences in which she omitted, substituted, or added a single word $(23 \%)$ or repetitions that differed more substantially in meaning from the target sentence (13\%).

4. Normal control subjects had no difficulty repeating the Spanish words on the first attempt. Anglicized pronunciations of the Spanish words were produced by the experimenter and accepted from the subjects.

5. Recent evidence we have obtained from another brain-damaged patient indicates that the short-term retention of lexical and semantic information may be disrupted independently of the ability to retain phonological information (Martin et al., 1992; Shelton et al., 1992).

6. Because of the focus of this review and space limitations, other neuropsychological findings relevant to short-term memory have not been touched upon. An important set of findings that are missing concern the role of phonological short-term memory in speech production and whether there needs to be a separation between an input phonological store and an output phonological store. Papers by Howard and Franklin (1990), Monsell (1987), and Romani (1992) address these issues.

(Manuscript received April 10, 1992; revision accepted for publication October 21, 1992.) 